\title{
gु
}

\section{Dynamics of a Two-Dimensional Quantum Spin Liquid: Signatures of Emergent Majorana Fermions and Fluxes}

\author{
J. Knolle, ${ }^{1, *}$ D. L. Kovrizhin, ${ }^{1,2}$ J. T. Chalker, ${ }^{3}$ and R. Moessner ${ }^{1}$ \\ ${ }^{1}$ Max Planck Institute for the Physics of Complex Systems, D-01187 Dresden, Germany \\ ${ }^{2}$ T.C.M. Group, Cavendish Laboratory, J. J. Thomson Avenue, Cambridge CB3 OHE, United Kingdom \\ and Imperial College London, London SW7 2AZ, United Kingdom \\ ${ }^{3}$ Theoretical Physics, Oxford University, 1, Keble Road, Oxford OX1 3NP, United Kingdom
}

(Received 3 February 2014; published 21 May 2014)

\begin{abstract}
We provide a complete and exact theoretical study of the dynamical structure factor of a twodimensional quantum spin liquid in gapless and gapped phases, as realized in Kitaev's honeycomb model. We show that there are direct signatures - qualitative and quantitative-of the Majorana fermions and gauge fluxes emerging in this model. These include counterintuitive manifestations of quantum number fractionalization, such as a neutron scattering response with a gap even in the presence of gapless excitations, and a sharp component despite the fractionalization of electron spin. Our analysis identifies new varieties of the venerable $x$-ray edge problem and explores connections to the physics of quantum quenches.
\end{abstract}

DOI: 10.1103/PhysRevLett.112.207203

Topological states of matter present a wide variety of striking new phenomena. Prominent among these is the fractionalization of electrons into unusual particles: Majorana fermions [1], Laughlin quasiparticles [2], or magnetic monopoles [3]. Their detection, however, is fundamentally complicated by the lack of any local order, such as, for example, the magnetization in a ferromagnet. While there are now several instances of candidate topological spin liquids [4], their identification remains challenging [5]. The study of spin liquids has been central to advancing our understanding of correlated phases of quantum matter ever since Anderson's proposal of the resonating valence bond liquid state [6], which provided, via the detour of high-temperature superconductivity, an early instance of a fractionalized topological state $[7,8]$. More recent manifestations hold the promise of realizing an architecture of quantum computing robust against decoherence [9].

Investigations of such topological states are hampered by the lack of suitable approaches, with numerical methods limited to small system sizes, to models with a robust excitation gap, or ones that avoid the sign problem in quantum Monte Carlo calculations. A benchmark is offered by the Kitaev model [1], which can be used as a representative example of an entire class of quantum spin liquids (QSL). While being a minimal model, it combines a raft of desirable features. First, it is described by a simple Hamiltonian involving only nearest-neighbor interactions on the honeycomb lattice (Fig. 1), by virtue of which it is a promising candidate for realization in materials physics [10], or in cold atom implementations of quantum simulators [11]. Second, it harbors two distinct topological quantum spin liquid phases, with either gapless or gapped
PACS numbers: 75.10.Kt, 75.40.Gb, 75.50.Mm, 78.70.Nx

Majorana fermion excitations. Finally, its solution can be reduced to the problem of Majorana fermions hopping in the background of an emergent static gauge field.

This remarkable feature permits, at least in principle, even an analysis of the model's dynamical properties, as noted in a seminal paper by Baskaran and co-workers [12], who pointed out an unexpected connection to the x-ray edge problem [13], results of which were used to extract asymptotic correlators of related models [14,15]. This problem, whose tour de force exact solution was obtained by Nozieres et al. [16], is one of the cornerstones of condensed matter physics, linked to the discovery of Anderson's "orthogonality catastrophe" [17] and a foundation for our understanding of local quantum quenches.
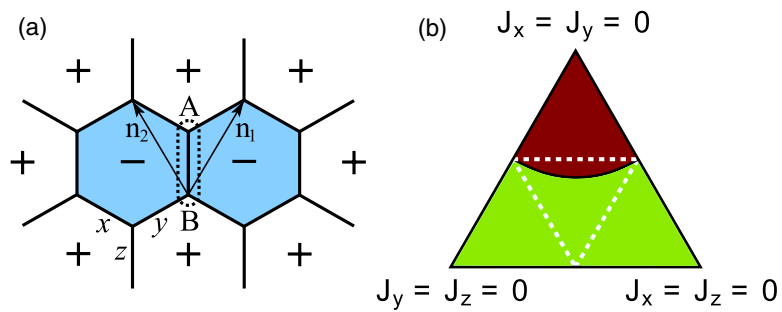

FIG. 1 (color online). (a) The structure of the model on the honeycomb lattice with two sublattices (labeled $A$ and $B$ ) and three bond directions (denoted $x, y, z$ ). The calculation of the dynamical response can be mapped to a local quantum quench, in which two adjoining $Z_{2}$ fluxes, shown in blue, are inserted. (b) The ground state and dynamical phase diagrams of the model: the phase with gapless fermion excitations fills the central triangle while gapped phases occupy the three outer triangles. The dynamical response $S^{z z}(\mathbf{q}, \omega)$ includes a contribution sharp in $\omega$ in the dark red region, but not in the light green region. 
The possibility of accessing dynamical properties of spin liquids is of particular importance as these contain information on fractionalized quasiparticles, and their theoretical study is topical in view of recent neutron scattering investigations of candidate QSL compounds [5,18,19]. Indeed, the $S=1 / 2$ spinons in the Heisenberg chain were most impressively visualized $[20,21]$ by an analysis of experiments based on the exact Bethe-ansatz solution, specific to one dimension. Our Letter provides this information in complete detail for the first time for a fractionalized quantum spin liquid in more than one dimension. Through the connections to quantum quenches and the physics of Majorana Fermions which appear in our discussion, it cements the central role played by the Kitaev model for our understanding of correlated and topological phases.

The model.-In the Kitaev model spin-half degrees of freedom at sites $j$ of a honeycomb lattice interact via nearest-neighbor Ising exchange $J_{a}$. Frustration and quantum fluctuations stem from linking the anisotropy direction $a=x, y, z$ in spin space to the bond direction in real space [Fig. 1(a)], a form of spin-orbit coupling. With Pauli matrices $\hat{\sigma}_{j}^{a}$ and using $\langle i j\rangle_{a}$ to indicate two sites sharing an $a$ bond, the Hamiltonian is

$$
H=-J_{x} \sum_{\langle i j\rangle_{x}} \hat{\sigma}_{i}^{x} \hat{\sigma}_{j}^{x}-J_{y} \sum_{\langle i j\rangle_{y}} \hat{\sigma}_{i}^{y} \hat{\sigma}_{j}^{y}-J_{z} \sum_{\langle i j\rangle_{z}} \hat{\sigma}_{i}^{z} \hat{\sigma}_{j}^{z}
$$

The ground states of Eq. (1) fall into two classes [1] gapped and gapless spin liquids-depending on the relative values of the $J_{a}$ [Fig. 1(b)]. Their emergent independent degrees of freedom are static $\mathbb{Z}_{2}$ gauge fluxes threading the plaquettes of the honeycomb lattice and Majorana fermions that hop between sites in this gauge field.

The model is solved [1] by introducing four Majorana fermions $\hat{c}_{i}, \hat{b}_{i}^{x}, \hat{b}_{i}^{y}, \hat{b}_{i}^{z}$ at each site and representing spins as $\hat{\sigma}_{i}^{a}=i \hat{c}_{i} \hat{b}_{i}^{a}$. Taking $\mathbf{r}$ as a unit cell coordinate, the Majorana fermions can be combined into two complex species: bond fermions $\hat{\chi}_{\langle i j\rangle_{a}}^{\dagger}=\frac{1}{2}\left(\hat{b}_{i}^{a}-i \hat{b}_{j}^{a}\right)$ and matter fermions $\hat{f}_{\mathbf{r}}=\frac{1}{2}\left(\hat{c}_{A \mathbf{r}}+i \hat{c}_{B \mathbf{r}}\right)$ [12]. Defining bond operators $\hat{u}_{\langle i j\rangle_{a}}=i \hat{b}_{i}^{a} \hat{b}_{j}^{a}$, which commute with $\hat{H}$, the model in terms of gauge degrees of freedom and Majorana fermions is

$$
\hat{H}=i \sum_{a,\langle i j\rangle_{a}} J_{a} \hat{u}_{\langle i j\rangle_{a}} \hat{c}_{i} \hat{c}_{j}
$$

The Hamiltonian $\hat{H}$ has the Bogoliubov-de Gennes form when expressed in terms of matter fermion operators $\hat{f}_{\mathbf{r}}^{\dagger}$ and $\hat{f}_{\mathbf{r}}$ for eigenstates of the gauge fermion operators $\hat{u}_{\langle i j\rangle}$. It, therefore, conserves fermion parity, but not fermion number. These features differentiate our spin dynamics problem from the conventional $\mathrm{x}$-ray edge problems and turn out to be central to our findings.
The Hilbert space of the Hamiltonian in Eq. (2) can now be decomposed into gauge $|F\rangle$ and matter $|M\rangle$ sectors, and we denote the ground state of $\hat{H}$ by $|0\rangle=\left|F_{0}\right\rangle \otimes\left|M_{0}\right\rangle$, in which $\hat{u}_{\langle i j\rangle_{a}}\left|F_{0}\right\rangle=+1\left|F_{0}\right\rangle$ for all bonds and $\left|M_{0}\right\rangle$ is the corresponding ground state of the Majorana hopping problem [1], whose Hamiltonian $\hat{H}_{0}$ is obtained from $\hat{H}$ by substituting all $\hat{u}_{\langle i j\rangle_{a}}$ with their ground-state eigenvalues +1 .

The dynamical structure factor--Our objective is to calculate the spin correlation function $S_{i j}^{a b}(t)=$ $\left\langle 0\left|\hat{\sigma}_{i}^{a}(t) \hat{\sigma}_{j}^{b}(0)\right| 0\right\rangle$ and its Fourier transform in space and time, the dynamical structure factor $S^{a b}(\mathbf{q}, \omega)$. The latter is proportional to the cross section obtained in an inelastic neutron scattering (INS) experiment, and at $\mathbf{q}=0$ to the signal obtained in electron spin resonance.

The measurement process creates a spin flip, which introduces a pair of fluxes in adjacent plaquettes (as illustrated in Fig. 1) and initiates the dynamical rearrangement of matter fermions in the modified gauge field. Because fluxes are static, site off diagonal spin correlations vanish except for $a$ components of a nearest-neighbor pair $\langle i j\rangle_{a}[12]$. We indicate this using the symbol $\delta_{\langle i j\rangle, a}$. (In the rest of the Letter, we show expressions for the nearest neighbor correlator; the ones for the site-diagonal terms are similar.) Crucially, the nonzero contributions to the structure factor can be expressed purely in terms of matter fermions in the ground state flux sector, subject to a perturbation $\hat{V}_{a}=-2 i J_{a} \hat{c}_{i} \hat{c}_{j}$, using the expression [12]

$$
S_{i j}^{a b}(t)=-i\left\langle M_{0}\left|e^{i \hat{H}_{0} t} \hat{c}_{i} e^{-i\left(\hat{H}_{0}+\hat{V}_{a}\right) t} \hat{c}_{j}\right| M_{0}\right\rangle \delta_{a b} \delta_{\langle i j\rangle, a} .
$$

The Hamiltonians $\hat{H}_{a}=\hat{H}_{0}+\hat{V}_{a}$ and $\hat{H}_{0}$ differ only in the sign of the Majorana hopping on the $a$ bond, representing insertion of the flux pair. The Lehmann representation of Eq. (3) can be written in the basis of many-body eigenstates of the Hamiltonian $\hat{H}_{a}$, denoted by $|\lambda\rangle$ with the corresponding energies $E_{\lambda}$, taking $E_{0}$ as the ground state energy of $\hat{H}_{0}$. We choose to work in the fixed gauge in which the eigenvalues of $\hat{u}_{\langle k l\rangle}$ are +1 for all bonds except the one linking the pair of sites $i$ and $j$ that appear in the correlator $S_{i j}^{a b}(t)$. Then

$$
\begin{aligned}
S_{i j}^{a b}(\omega)= & -i \sum_{\lambda}\left\langle M_{0}\left|\hat{c}_{i}\right| \lambda\right\rangle\left\langle\lambda\left|\hat{c}_{j}\right| M_{0}\right\rangle \\
& \times \delta\left[\omega-\left(E_{\lambda}-E_{0}\right)\right] \delta_{\langle i j\rangle, a} \delta_{a b} .
\end{aligned}
$$

Results.-We start our discussion of the results displayed in Figs. 2 and 3 by explaining the salient qualitative features in terms of the selection rules imposed by the fractionalization of the electrons into fluxes and Majoranas. It is instructive to do so using Eq. (4), from which the central aspects of the response can be read off, and we relegate an explanation of the numerically exact solution of Eq. (3) which is used to obtain the results in Figs. 2 and 3 to the Supplemental Material [22]. 

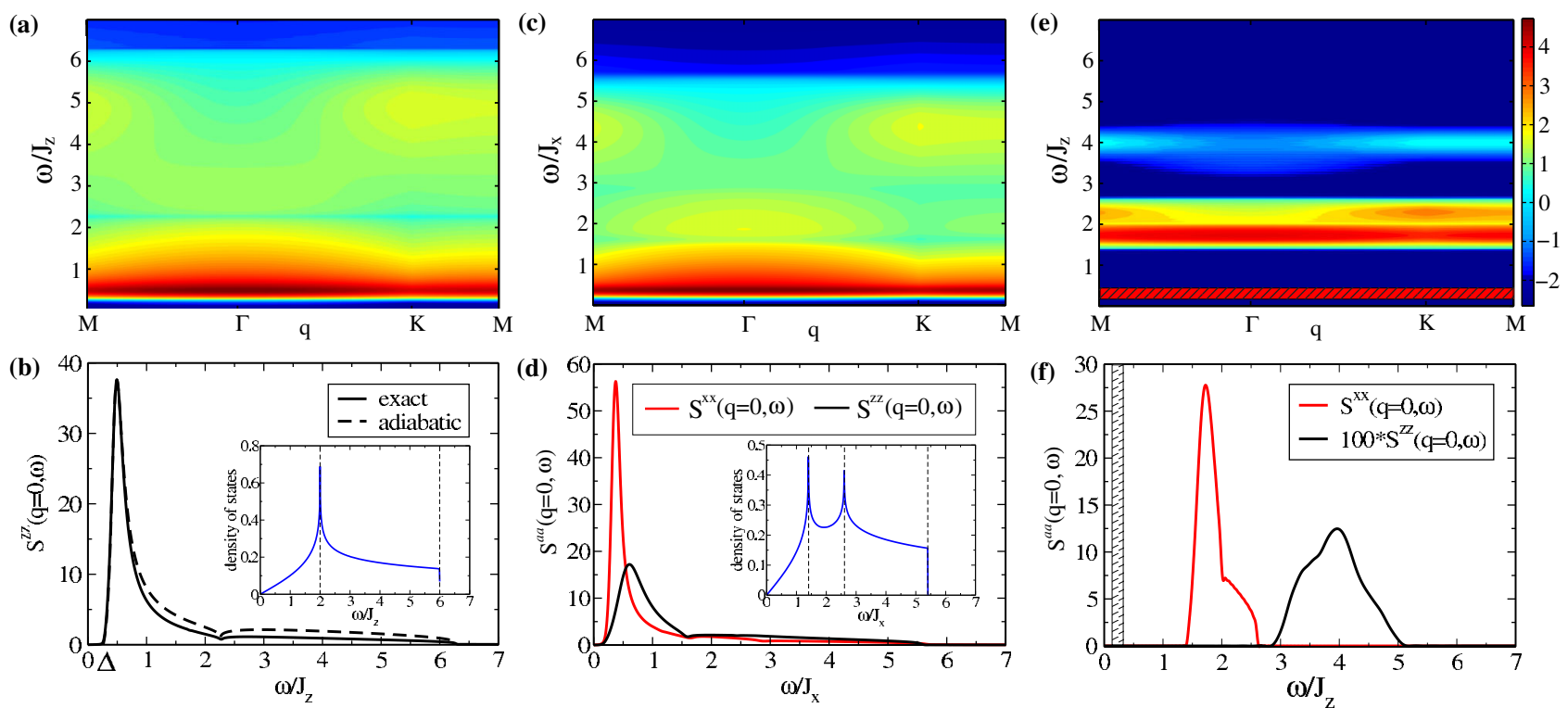

FIG. 2 (color online). Total dynamical structure factor $S(\mathbf{q}, \omega)=\sum_{a} S^{a a}(\mathbf{q}, \omega)$ and the inequivalent components $S^{a a}(0, \omega)$, as would be measured in inelastic neutron scattering and electron spin resonance, respectively, evaluated for three points in the phase diagram: $(\mathrm{a}+\mathrm{b})$ the symmetric point $\left(J_{x}=J_{y}=J_{z}\right) ;(\mathrm{c}+\mathrm{d})$ a gapless asymmetric point $\left(J_{x}=J_{y}, J_{z}=0.7 J_{x}\right)$; and $(\mathrm{e}+\mathrm{f})$ a gapped point $\left(J_{x}=J_{y}=0.15 J_{z}\right)$. Top: $S(\mathbf{q}, \omega)$ on a logarithmic color scale as a function of $\omega$ along the cut MГKM through the Brillouin zone. Bottom: dynamical susceptibility $S^{a a}(0, \omega)$ for $a=z, x$ at the same values of the exchange. Comparison with the adiabatic response as explained in the main text is given in panel b (black dashed line). The dashed line in (f) indicates the delta-function contribution to the response, present only in the region of the dynamical phase diagram colored red in Fig. 1(b). The insets to panels (b) and (d) show the density of states of the matter fermions.

First, in both gapped and gapless phases, response vanishes below the two-flux gap $\Delta=E_{\lambda_{0}}-E_{0}$, the difference between the ground state energies in a system with and without the flux pair. (At the isotropic point, $\Delta \simeq 0.26 \mathrm{~J}$ [1].) It is remarkable that in an INS experiment, the response of a gapless QSL will show an excitation gap which is directly related to the emergent gauge field. A similar gap was found in a modified Kitaev model with a Fermi circle excitation spectrum [14].

Above the gap $\Delta$, the response thus reflects the physics of the matter sector. Here, our analysis uncovers an entirely new structure in the phase diagram of the Kitaev model, Fig. 1(b). Specifically, an important consequence of the fact that $\hat{H}$ in Eq. (2) conserves matter fermion parity is that the nonzero contributions to Eq. (4) come only from excited states $|\lambda\rangle$ with parity opposite to the ground state $\left|M_{0}\right\rangle$. As a result, two distinctively different alternatives arise: either (I) the ground states $\left|M_{0}\right\rangle$ of $\hat{H}_{0}$ and $\left|\lambda_{0}\right\rangle$ of $\hat{H}_{a}$ have the same parity, in which case, the states $|\lambda\rangle$ must contain an odd number of excitations, or (II) the ground states have opposite parity and $|\lambda\rangle$ contains an even number of excitations. For (II), the sector with zero excitations is an important special case, distinct from the sectors with two, four, or more excitations because it contains only a single state: the ground state of $H_{a}$. Its contribution to $S_{i j}^{a b}(\omega)$ is therefore sharp in $\omega$, whereas the contributions from sectors with nonzero excitation numbers are broad.
To determine the dynamical phase boundary separating the regime (II) that has a sharp response from the region (I) that does not, we calculate numerically the ground state overlap $\left|\left\langle\lambda_{0} \mid M_{0}\right\rangle\right|$ (see [22], for details), to find whether both have the same parity (nonzero overlap) or opposite

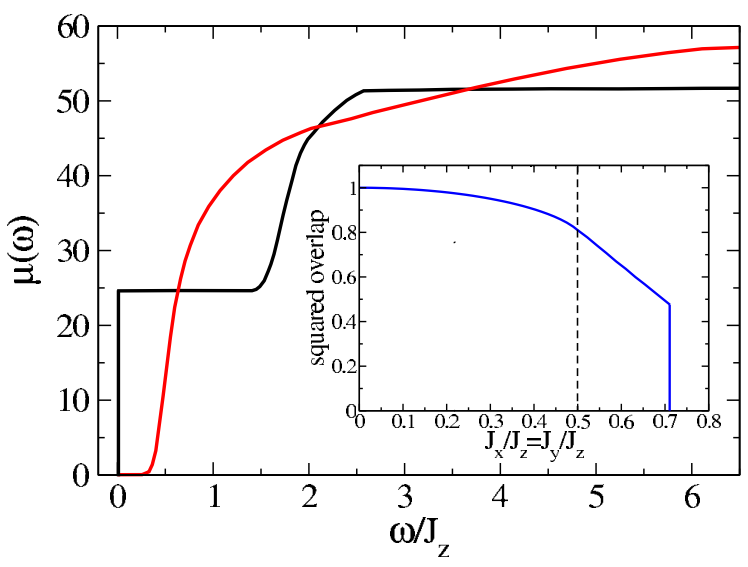

FIG. 3 (color online). The integrated structure factor $\mu(\omega)=$ $\int_{0}^{\omega} d \omega^{\prime} \sum_{a} S^{a a}\left(0, \omega^{\prime}\right)$ in the gapless $J_{x}=J_{y}=J_{z}$ (red) and the gapped $J_{x} / J_{z}=J_{y} / J_{z}=0.15$ (black) phases. The sharp step in the black curve at low $\omega$ at the two-flux gap $\Delta \simeq J_{x}^{4} / 8 J_{z}^{3}$ is due to the $\delta$-function contribution of the strong bond correlator $(a=z)$. Inset: the dependence of the ground state overlap $\left|\left\langle\tilde{\lambda}_{0} \mid M_{0}\right\rangle\right|^{2}$, proportional to the $\delta$-function contribution, on interaction strength, along the line $J_{x} / J_{z}=J_{y} / J_{z}$, see Supplemental Material [22] for details. 
parity (zero overlap). The results are illustrated in Fig. 1(b). The reason why the two ground states may have opposite parity is most transparent deep in the gapped phase, e.g., $J_{x}, J_{y} \ll J_{z}$. There, each $z$ bond can be occupied or empty with a single complex fermion, and the ground state of the system without a flux has an even number of those. Addition of the flux pair amounts to flipping the sign of $J_{z}$, hence, inverting the energy of that Fermion. The ground state with flux thence has opposite parity, as its energy is lowered by occupying the previously empty state.

For (I) [Figs. 2(a)-2(d)], single particle excitations dominate the response, which is broad in energy, so that its amplitude is appreciable only within the matter fermion bandwidth. Indeed, only about $2.5 \%$ of the signal at the symmetric point arises from multiparticle contributions (see Supplemental Material [22]) in stark contrast to the case of the Heisenberg chain [20], where the corresponding number is almost $30 \%$.

For (II) [Figs. 2(e) and 2(f)], in striking opposition, the response includes a finite-weight $\delta$-function component in $\omega$ at the difference $\Delta$ in ground state energies, since the corresponding matrix element is finite. It is a remarkable and unexpected finding that-despite fractionalization-the INS response has a component sharp in energy [displayed in Fig. 3(b)]. Note that the location in the phase diagram of the dynamical transition at which this sharp response appears is distinct from the ground state phase boundary: it lies entirely within the gapless phase [Fig. 1(b)].

Discussion.-Formally, Eq. (3) represents an example of a quantum quench: it involves the overlap between a state $\left\langle M_{0}\right| \hat{c}_{i}$ that is simple in terms of $\hat{H}_{0}$ (a superposition of single-particle excitations) and a similar state $\hat{c}_{j}\left|M_{0}\right\rangle$ after the latter has evolved for time $t$ under a different Hamiltonian $\hat{H}_{a}$. The broad features of the resulting response of the Majorana fermions above $\Delta$ are a result of this quench. Quite surprisingly, this can be well approximated by replacing the instantaneous flip of the bond by an adiabatic, rather than sudden, switching on of the potential $\hat{V}_{a}$. This amounts to replacing $\left|M_{0}\right\rangle$ in Eq. (4) by the Majorana ground state in the presence of the fluxes. One can show that in the limit of low energies, the matter fermion eigenstates are, in fact, insensitive to the flux addition, so that the resulting approximation [dashed line, Fig. 2(b)] becomes exact as $\omega$ approaches $\Delta$.

It is interesting to compare the energy dependence of the structure factor with the density of states for matter fermions [Figs. 2(b) and 2(d)]. Response is substantial over the entire single-particle bandwidth (shifted in energy by $\Delta$ ), with linear onset above the gap. However, as a qualitative signature of the effect of gauge fluxes on matter fermion dynamics, the response is far from being simply proportional to the density of states. Instead, the peak in the latter at $2 J_{z}$ due to the van Hove singularity [see inset to Fig. 2(b)] yields a dip in the response. Away from the symmetric point, there are two van Hove singularities in the density of states, and, in addition, there is a distinct response for different spin components, showing one or two minima in the corresponding dynamical susceptibility [Fig. 2(d)].

Despite the formal similarities between the time-dependent correlator Eq. (3) and the x-ray edge problem, the physics arising from it is quite different. First, depending on the exchange $J_{a}$ one must study a local quantum quench in either gapless or gapped phases, the latter not presenting the possibility of low-energy fermionic excitations. Second, for inequivalent values of $J_{a}$, the correlators for different spin components are different. Third, the Majorana fermions in our calculation arise due to fractionalization of spin degrees of freedom as emergent particles. Fourth, they have not number, but only parity conservation, and their dispersion exhibits Dirac cones. Finally, the $\delta$-function response (Fig. 3 inset) is diametrically opposed to Anderson's orthogonality catastrophe, which would correspond to a vanishing signal at $\Delta$.

The Kitaev model is a representative of a class of $\mathbb{Z}_{2}$ spin liquids coupled to (gapped or gapless) Majorana fermions. The qualitative features described above should therefore be characteristic of this broad class of topological states. While a potential cold atom realization will likely harbor few perturbations to the Kitaev Hamiltonian Eq. (1), magnetic materials usually include other terms, as extensively discussed for Kitaev-Heisenberg models following Ref. [10]. Both the flux gap and the fermion parity underpinning our results are robust to such perturbations. Just as in the analogous case of the Heisenberg chain [20], where integrability is imperfect in reality but all qualitative features are well-observed experimentally, so we similarly expect quantitative changes such as a small degree of smearing out of the $\delta$-function response or a more gradual onset of the signal around $\Delta$. Crucially, the central features we have discovered will be visible as fingerprints betraying the presence of fractionalized Majorana fermions and emergent gauge fluxes in INS and electron spin resonance experiments.

We thank F. H. L. Essler and G. Baskaran for helpful discussions. J. K. acknowledges support from the Studienstiftung des deutschen Volkes, the IMPRS Dynamical Processes in Atoms, Molecules, and Solids, DFG within GRK1621. J. T.C. is supported in part by EPSRC Grant No. EP/I032487/1. D. K. is supported by EPSRC Grants No. EP/J017639/1 and No. EP/J009636/1, and acknowledges the GGI workshop "New Quantum States of Matter In and Out of Equilibrium", where part of this work was done. This collaboration was supported by the Helmholtz Virtual Institute "New States of Matter and their Excitations".

*jknolle@pks.mpg.de

[1] A. Yu. Kitaev, Ann. Phys. (Amsterdam) 321, 2 (2006).

[2] R. B. Laughlin, Phys. Rev. Lett. 50, 1395 (1983). 
[3] C. Castelnovo, R. Moessner, and S. L. Sondhi, Nature (London) 451, 42 (2008).

[4] P. A. Lee, Science 321, 1306 (2008).

[5] T. H. Han, J. S. Helton, S. Chu, D. G. Nocera, J. A. Rodriguez-Rivera, C. Broholm, and Y.S. Lee, Nature (London) 492, 406 (2012).

[6] P. W. Anderson, Mater. Res. Bull. 8, 153 (1973).

[7] R. Moessner and S. L. Sondhi, Phys. Rev. Lett. 86, 1881 (2001).

[8] D. S. Rokhsar and S. A. Kivelson, Phys. Rev. Lett. 61, 2376 (1988).

[9] C. Nayak, S. H. Simon, A. Stern, M. Freedman, and S. Das Sarma, Rev. Mod. Phys. 80, 1083 (2008).

[10] G. Jackeli and G. Khaliullin, Phys. Rev. Lett. 102, 017205 (2009).

[11] L. M. Duan, E. Demler, and M. D. Lukin, Phys. Rev. Lett. 91, 090402 (2003).

[12] G. Baskaran, S. Mandal, and R. Shankar, Phys. Rev. Lett. 98, 247201 (2007).

[13] A. O. Gogolin, A. A. Nersesyan, and A. M. Tsvelik, Bosonization and Strongly Correlated Systems (Cambridge University Press, Cambridge, England, 2004).

[14] K. S. Tikhonov and M. V. Feigelman, Phys. Rev. Lett. 105, 067207 (2010).

[15] K. S. Tikhonov, M. V. Feigelman, and A. Yu. Kitaev, Phys. Rev. Lett. 106, 067203 (2011).
[16] P. Nozieres and C. T. De Dominicis, Phys. Rev. 178, 1097 (1969).

[17] P. W. Anderson, Phys. Rev. Lett. 18, 1049 (1967).

[18] S. K. Choi et al., Phys. Rev. Lett. 108, 127204 (2012).

[19] Y. Singh, S. Manni, J. Reuther, T. Berlijn, R. Thomale, W. Ku, S. Trebst, and P. Gegenwart, Phys. Rev. Lett. 108, 127203 (2012).

[20] M. Mourigal, M. Enderle, A. Klöpperpieper, J.-S. Caux, A. Stunault, and H. M. Rønnow, Nat. Phys. 9, 435 (2013).

[21] D. A. Tennant, R. A. Cowley, S. E. Nagler, and A. M. Tsvelik, Phys. Rev. B 52, 13368 (1995).

[22] See Supplemental Material at http://link.aps.org/ supplemental/10.1103/PhysRevLett.112.207203 of the numerically exact solution, which includes Refs. [23-26].

[23] A. A. Abrikosov, L. P. Gorkov, and I. E. Dzyaloshinski, Methods of Quantum Field Theory in Statistical Physics (Dover Publications, Mineola, 1975).

[24] N. I. Muskhelishvili, Singular Integral Equations: Boundary Problems of Function Theory and Their Application to Mathematical Physics (Dover Publications, Mineola, 2008).

[25] V. I. Grebennikov, Y. U. Babanov, and O. B. Sokolov, Phys. Status Solidi (b) 79, 423 (1977).

[26] J. P. Blaizot and G. Ripka, Quantum Theory of Finite Systems (MIT Press, Cambridge, 1985). 